Check for updates

Cite this: Mater. Adv., 2021, 2,4743

Received 30th April 2021 Accepted 3rd June 2021

DOI: $10.1039 / \mathrm{d} 1 \mathrm{ma} 00395$

rsc.li/materials-advances

\title{
Regulating the Bi NIR luminescence behaviours in fluorine and nitrogen co-doped germanate glasses
}

\author{
Fuguang Chen, ${ }^{a}$ Yafei Wang, ${ }^{a}$ Weiwei Chen, ${ }^{a}$ Puxian Xiong, ${ }^{\mathrm{b}}$ Bofan Jiang, ${ }^{\mathrm{a}}$ \\ Shifeng Zhou, ${ }^{a}$ Zhijun Ma (iD *a and Mingying Peng (D) ab
}

\begin{abstract}
Bi-Doped nitridated germanate glasses covering the whole NIR region from 800-1600 nm have attracted considerable attention due to their potential application in tunable fiber lasers and optical amplifiers. However, it remains challenging to regulate the luminescence behaviour of Bi because of the coexistence of multi-centers in the glasses. Here, we successfully regulated multi Bi NIR centers to obtain a flatter and ultrabroadband emission in nitridated germanate glasses. By varying the alkaline earth metal fluorides from $\mathrm{MgF}_{2}$ to $\mathrm{BaF}_{2}$, the glass structures were gradually depolymerized. A looser glass network, on one hand, promoted $\mathrm{Bi}$ in lower valences to be oxidized into $\mathrm{Bi}^{2+}$ and $\mathrm{Bi}^{3+}$, thus reducing the NIR emission intensity at $\sim 1150$ and $\sim 1260 \mathrm{~nm}$; on the other hand, it also enhanced the energy transfer from $\mathrm{Bi}^{+}$to $\mathrm{Bi}^{0}$, which resulted in a relatively flatter emission band. In addition, the depolymerised glass structure could facilitate more nitride bonds, which are favorable for dispersing and stabilizing the new Bi NIR active centers related to germanate for $\sim 930$ and $\sim 1490 \mathrm{~nm}$ emission. This investigation offers an efficient way to manipulate the multi-center luminescence behaviour of $\mathrm{Bi}$ in nitridated germanate glasses, and is beneficial to understand the mechanism of Bi NIR emission centers in glasses.
\end{abstract}

\section{Introduction}

The arrival of the 5th generation of communication technology (5G) is often accompanied by the explosive growth of information and data, which require bigger information transmission capacity for the current optical communication system. One possible way to improve the performance of the optical communication system is to broaden the data transmission channels, thus increasing the information transmission capacity. Laser materials operating in the broadband NIR spectral region (1200-1600 nm) are the major components for developing modern communication systems with unprecedented performance. ${ }^{1-4}$ Due to the excellent fluorescence characteristics, rare-earth-doped materials, as a type of efficient gain medium, particularly near-infrared (NIR) optical amplifiers and tunable lasers, have attracted widespread attention. Unfortunately, the $\mathrm{f}-\mathrm{f}$ electronic transition, which is shielded by the

\footnotetext{
${ }^{a}$ The China-Germany Research Center for Photonic Materials and Device, the State Key Laboratory of Luminescent Materials and Devices, and Guangdong Provincial Key Laboratory of Fiber Laser Materials and Applied Techniques, the School of Materials Science and Engineering, South China University of Technology, Guangzhou 510641, China. E-mail: zhijma@scut.edu.cn

${ }^{b}$ School of Physics and Optoelectronics, the State Key Laboratory of Luminescent Materials and Devices, and Guangdong Provincial Key Laboratory of Fiber Laser Materials and Applied Techniques, South China University of Technology,

Guangzhou 510641, China
}

outer-layer electrons, is almost unaffected by glass composition, making it difficult to modulate the emission bandwidth and peak position. As a result, the gain bandwidth of traditional rare-earth-doped materials has always been quite narrow (not exceeding $100 \mathrm{~nm}$ ), which cannot make full use of the ultra-lowattenuation telecommunication windows of silica glass fibers (1260-1675 nm)..$^{5-9}$ Therefore, it is urgent to explore new photonic materials with an ultra-broadband NIR emission and long lifetime as a gain medium for potential applications in broadband optical amplifiers and tunable lasers.

In the past decades, Bi-doped glasses and fibers have been confirmed to exhibit intriguing broad NIR photoemission from 1000 to $1600 \mathrm{~nm}$, which made them a promising medium for optical amplifiers and NIR lasers. ${ }^{10-21}$ Although a significant progress has now been made in improving the performance of Bi-doped glasses and fibers, the emission intensity of the widely used C and L bands (1530-1625 nm) is relatively weak. In recent years, Cao et al. realized a super-broad emission band shape, which extends over the whole NIR spectral region (850-1600 nm) via the topochemical reduction of bismuth in germanate glasses co-doped with $\mathrm{AlN}, \mathrm{Si}_{3} \mathrm{~N}_{4}$ or $\mathrm{SiC}^{20,22-24}$ The topochemical reduction in germanate glasses not only enhances the near-infrared luminescence of bismuth but also generates new NIR emission centers located at $\sim 930$ and $\sim 1500 \mathrm{~nm}$, which are related to germanium. Due to the coexistence of 
multiple $\mathrm{Bi}$ NIR activate centers (such as $\mathrm{Bi}^{+}, \mathrm{Bi}^{0}$ and $\mathrm{Bi}$ related to germanium) and their super sensitivity to the changes in the crystal field, it remains challenging to regulate the Bi NIR luminescence behaviour..$^{21,25-28}$

At present, Bi-doped fibers are mainly based on oxide glasses, which show superior chemical and optical stability; however, simultaneously, they suffer from the large phonon energy ( $\sim 1200 \mathrm{~cm}^{-1}$ for silica glass). Compared to oxide glasses, the phonon energy of a fluoride glass $\left(\sim 550 \mathrm{~cm}^{-1}\right.$ for ZBLAN glass) is relatively low, which helps to reduce the non-radiative transition and to enhance the luminescence, but its chemical stability is poor. Hence, oxyfluoride glasses combining the advantages of both oxide and fluoride glasses, may be one of the best candidates for optical materials. Owing to this reason, oxyfluoride glasses have been confirmed to be promising rare-earth-doped optical materials in recent years. ${ }^{29-31}$ In 2012 , Xu et al. reported that the substitution of magnesium fluoride for magnesium oxide in Bi-doped silicate glasses could significantly increase the intensity and lifetime of the NIR luminescence. ${ }^{32}$ In 2020, Chen et al. manifested that the partial substitution of barium fluoride for barium oxide in Bi-doped nitridated germanate glasses could facilitate the formation of nitride bonds to induce NIR luminescence in the glasses. ${ }^{33}$ However, to the best of our knowledge, there are a few reports related to a systematic study on different fluorides of alkali earth metals on the regulation of multi-center luminescence behaviour of $\mathrm{Bi}$ in nitridated germanate glasses.

In this study, taking topochemically reduced multicomponent germanate glasses as models, a series of nitridated germanate glasses co-doped with a low Bi content and different fluorides of alkaline earth metals were investigated. The results here indicated that the Bi NIR luminescence behaviour can be manipulated by varying the alkaline earth metal ions, and flat and tunable NIR emission spectra spanning from $850 \mathrm{~nm}$ to $1600 \mathrm{~nm}$ were obtained by adjusting the glass structures. We believe that the present work is beneficial to promoting the design of Bi-doped germanate glass fibres with a tunable and ultra-broadband NIR luminescence.

\section{Experimental section}

\subsection{Synthesis}

Glass composition with molar compositions of $76 \mathrm{GeO}_{2}-9 \mathrm{Al}_{2} \mathrm{O}_{3}-$ $x \mathrm{CaF}_{2}-(12-x) \mathrm{CaO}-3 \mathrm{AlN}-0.005 \mathrm{Bi}_{2} \mathrm{O}_{3}(x=0,4,6,8$, and 10) and $76 \mathrm{GeO}_{2}-9 \mathrm{Al}_{2} \mathrm{O}_{3}-6 \mathrm{MF}_{2}-6 \mathrm{BaO}-3 \mathrm{AlN}-0.005 \mathrm{Bi}_{2} \mathrm{O}_{3}(\mathrm{M}=\mathrm{Mg}, \mathrm{Ca}, \mathrm{Sr}$, and $\mathrm{Ba}$ ) were prepared by traditional melting and quenching methods. For convenience, the glass samples were denoted as $x \mathrm{CF}(x=0,4,6,8$, and 10) and $\mathrm{MF}, \mathrm{CF}, \mathrm{SF}, \mathrm{BF}$, respectively. Analytical grade $\mathrm{Al}_{2} \mathrm{O}_{3}, \mathrm{MgF}_{2}, \mathrm{CaF}_{2}, \mathrm{SrF}_{2}, \mathrm{BaF}_{2}, \mathrm{CaCO}_{3}, \mathrm{BaCO}_{3}$ and high-purity $\mathrm{GeO}_{2}$ (99.999\%), AlN and $\mathrm{Bi}_{2} \mathrm{O}_{3}$ (99.99\%) were used as the starting materials. Batches of raw materials (15 g) were mixed homogeneously in an agate mortar. Each batch of the mixture was melted in an alumina crucible at a temperature of $1520{ }^{\circ} \mathrm{C}$ in air for $30 \mathrm{~min}$. Afterwards, the melt was cast onto a stainless-steel plate, and finally pressed by another stainless-steel plate to speed up the cooling process and avoid crystallization. Finally, the glasses were cut and polished for optical analysis.

\subsection{Characterization}

Optical absorption spectra of the samples were recorded using a PerkinElmer Lambda 900 UV/Vis spectrophotometer over the spectral range of 300-1000 $\mathrm{nm}$. Static excitation and emission spectra, dynamic emission spectra excited at $460 \mathrm{~nm}$, and luminescence lifetime were obtained using an Edinburgh FLS920 spectrofluorometer equipped with a continuous wave $450 \mathrm{~W}$ Xe lamp and a microsecond flashlamp ( $\mu$ F900) as excitation sources. Fourier transform infrared (FT-IR) spectra were measured in the transmission mode using a Bruker Vector 33 spectrometer by dispersing glass powders into $\mathrm{KBr}$ pellets. Electron paramagnetic resonance (EPR) spectra were collected using an X-band spectrometer (Bruker E500). All measurements were performed at room temperature.

\section{Results and discussion}

In order to verify that the introduction of an appropriate amount of fluorine in the glasses is beneficial to improve the NIR luminescence property of $\mathrm{Bi}$, a series of Bi-doped germanate glasses containing different contents of fluorine were investigated. Fig. 1 illustrates the emission spectra of $76 \mathrm{GeO}_{2}-9 \mathrm{Al}_{2} \mathrm{O}_{3}-x \mathrm{CaF}_{2}-$ $(12-x) \mathrm{CaO}-3 \mathrm{AlN}-0.005 \mathrm{Bi}_{2} \mathrm{O}_{3}(x=0,4,6,8$, and 10). The intensities of the emission bands at $\sim 920 \mathrm{~nm}$ and $\sim 1484 \mathrm{~nm}$ first increase, and then, decease with the further increase in the fluorine content. However, the intensity of the emission band at $\sim 1150 \mathrm{~nm}$ kept decreasing continuously. As shown in the inset of Fig. 1, the intensities of the emission bands at $\sim 920$ and $\sim 1484 \mathrm{~nm}$ reached the appropriate state at $4 \mathrm{~mol} \%$. The intensity of the emission band at $\sim 1500 \mathrm{~nm}$ decreases continuously, which indicates that fluorination can effectively manipulate the Bi NIR emission bands, and enhance the emission intensity, particularly for the emission bands at $\sim 920$ and $\sim 1484 \mathrm{~nm}$.

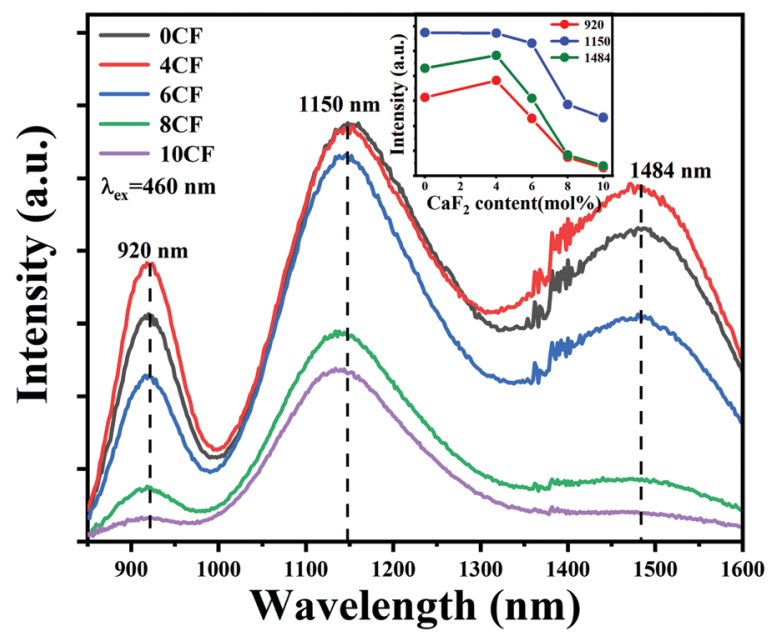

Fig. 1 The emission spectra $\left(\lambda_{\mathrm{ex}}=460 \mathrm{~nm}\right)$ of the $x \mathrm{CF}(x=0,4,6,8$, and 10) glass samples. The inset is the integral intensity of the $\sim 920, \sim 1150$, and $\sim 1484 \mathrm{~nm}$ emission bands as a function of the content of fluoride. 
When we introduced different fluorides of alkaline earth metals to Bi-doped nitridated germanate glasses, the luminescence spectral configuration of the glasses could be tuned. As presented in Fig. 2(a), under an excitation at $460 \mathrm{~nm}$, an ultrabroadband NIR luminescence covering $850-1600 \mathrm{~nm}$ with three predominant peaks at $\sim 924, \sim 1150$ and $\sim 1496 \mathrm{~nm}$ related to the Bi NIR centers is observed in all samples. According to previous reports, the emission bands at $\sim 924$ and $1496 \mathrm{~nm}$ can be attributed to the new Bi-activated centers generated by the addition of nitride in germanate glassses, ${ }^{22,23,34}$ while the emission band at $\sim 1150 \mathrm{~nm}$ originated from the typical transition ${ }^{3} \mathrm{P}_{1} \rightarrow{ }^{3} \mathrm{P}_{0}$ of $\mathrm{Bi}^{+} .{ }^{20,35}$ Moreover, as shown in Fig. 2(a), the integral intensity of the $\sim 1150 \mathrm{~nm}$ emission band showed a dramatical decreasing trend as the radius of alkaline earth metal ion increased, while the integral intensity of the $\sim 924$ and $\sim 1496 \mathrm{~nm}$ emission bands got slightly enhanced. Moreover, different from the $\sim 1150 \mathrm{~nm}$ emission band, the emission bands located at $\sim 924$ and $\sim 1496 \mathrm{~nm}$ demonstrate an increasing trend, and the shapes of their emission bands are similar, as indicated by the corresponding normalized emission spectra (to the intensity at $\sim 1150 \mathrm{~nm}$ ) in Fig. 2(b). The above phenomenon indicates that the Bi-activated NIR centers responsible for $\sim 924, \sim 1150$, and $\sim 1496 \mathrm{~nm}$ responded differently to the change of alkaline earth metal fluorides.
More importantly, the FWHM of the $\sim 1150 \mathrm{~nm}$ emission band increased from 187 to $443 \mathrm{~nm}$ by varying the alkaline earth metals from $\mathrm{Mg}$ to Ba. Although the overall emission intensity at $\sim 1150 \mathrm{~nm}$ shows a weakening trend, a new emission band appeared at $\sim 1260 \mathrm{~nm}$, which is due to the typical transition ${ }^{2} \mathrm{D}_{3 / 2} \rightarrow{ }^{4} \mathrm{~S}_{3 / 2}$ of $\mathrm{Bi}^{0}$, and emerged gradually in the $\mathrm{SF}$ and $\mathrm{BF}$ glass samples (Fig. 2(b)). ${ }^{18}$ Owing to the strong overlap between the $\sim 1150$ and $\sim 1260 \mathrm{~nm}$ emission bands, it was hard to detect the $\sim 1260 \mathrm{~nm}$ band in the MF and CF glass samples. A Gauss fitting of the emission spectra of the four samples was conducted towards the emission spectra of the glasses with different fluorides of alkaline earth metals. As shown in Fig. 2(c), there are four emission bands in each sample. Although the overall emission intensity at $\sim 1150 \mathrm{~nm}$ decreased, the ratio between the emission intensity of $\sim 1260 \mathrm{~nm}$ to that of $\sim 1150 \mathrm{~nm}$ increased with the increase in the radius of alkaline earth metal ions (Fig. 2(d)). This result was slightly different from the phenomenon reported by a previous investigation, in which only a redshift and an intensity decrease occurred as the radius of alkaline earth metal ions increased. ${ }^{36-38}$ Here, a tentative reason to explain this phenomenon is that the energy transfer between $\mathrm{Bi}^{+}$and $\mathrm{Bi}^{0}$ occurred.

The absorption spectra of the glasses are shown in Fig. 3. These samples all exhibited three absorption peaks centered at
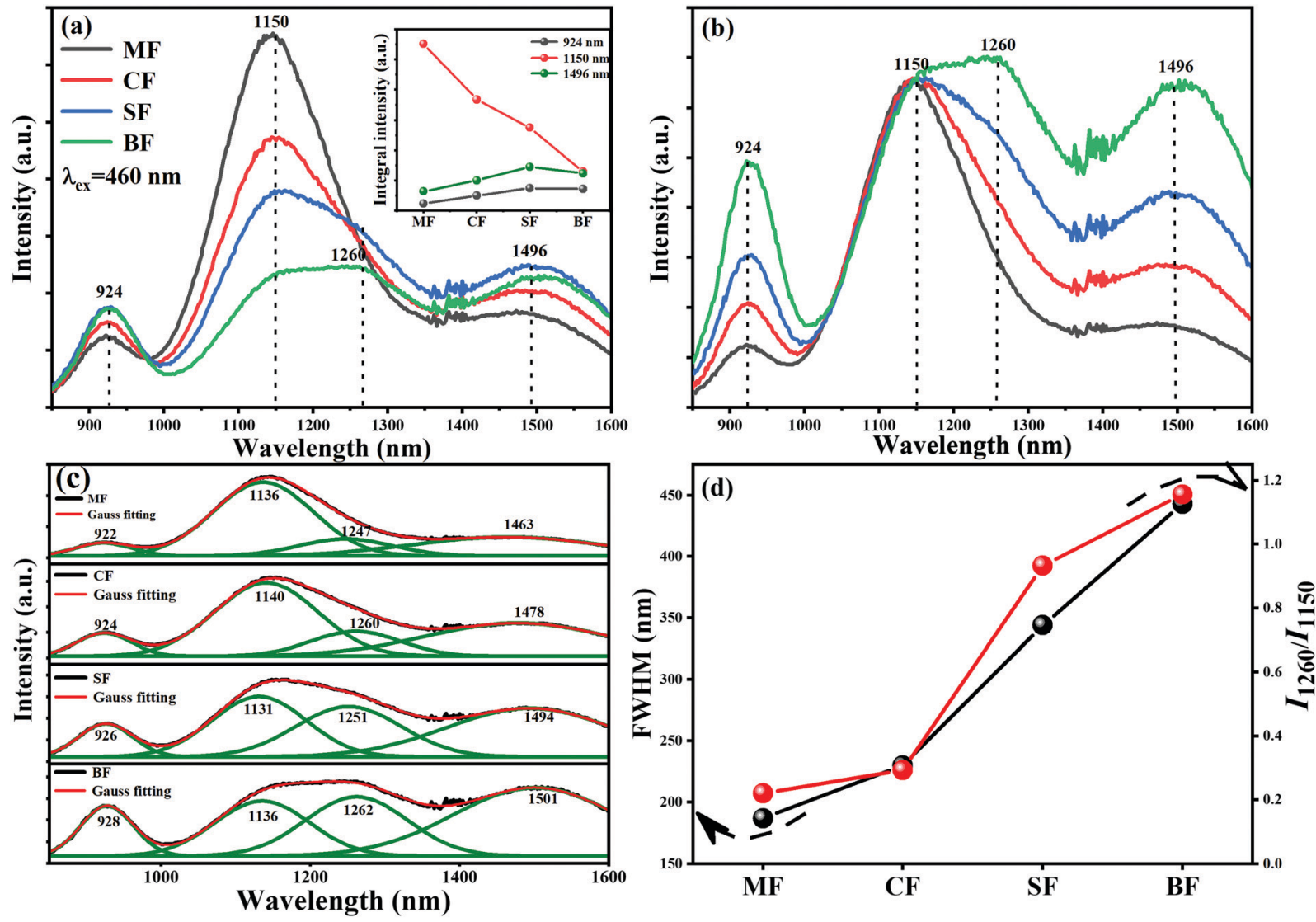

Fig. 2 (a) Emission spectra $\left(\lambda_{\mathrm{ex}}=460 \mathrm{~nm}\right.$ ) of the MF, CF, SF, BF glass samples. The inset is the integral intensity of the $\sim 924, \sim 1150, \sim 1260 \mathrm{~nm}$ and $\sim 1496 \mathrm{~nm}$ emission bands as a function of alkaline earth metal fluorides. (b) Corresponding emission spectra normalized to the emission peak at $\sim 1150 \mathrm{~nm}$. (c) Comparison between the emission spectra of sample MF, CF, SF, BF by Gauss fitting. (d) Dependence of the FWHM of the $\sim 1150 \mathrm{~nm}$ emission band and the ratio of $I_{1260} / I_{1150}$ on the alkaline earth metal fluorides. 


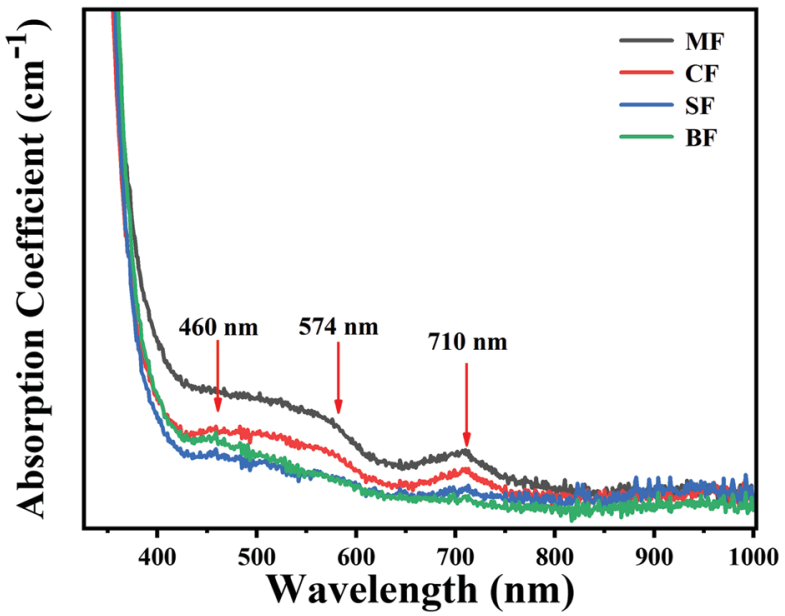

Fig. 3 The optical absorption spectra of MF, CF, SF, BF glass samples.

$\sim 460, \sim 574$ and $\sim 710 \mathrm{~nm}$, respectively. It is widely accepted that the $\sim 460 \mathrm{~nm}$ absorption band is attributed to the typical absorption of $\mathrm{Bi}^{0}$, while the $\sim 710 \mathrm{~nm}$ band is from $\mathrm{Bi}^{+} .^{26,27,37}$ However, as compared to the $\sim 460$ and $710 \mathrm{~nm}$ bands, the $\sim 574 \mathrm{~nm}$ band presented a flatter and wider shape, which may be caused by the new Bi-activated NIR centers. By varying alkaline earth metal fluorides from $\mathrm{MgF}_{2}$ to $\mathrm{BaF}_{2}$, all the Bi-related absorption intensities gradually decreased.

To obtain more information on the Bi-related centers, the excitation spectrum of different emission bands of the BF glass is collected, as shown in Fig. 4. It can be seen that the four NIR emission bands could be well excited by blue light, which shows a great potential as a blue LED-excited broadband NIR light source. The four excitation spectra, however, are different in some details. When monitored at $930 \mathrm{~nm}$, five excitation bands at $\sim 338, \sim 394, \sim 456, \sim 574, \sim 672$ and $\sim 800 \mathrm{~nm}$ are observed. These complicated excitation features are different

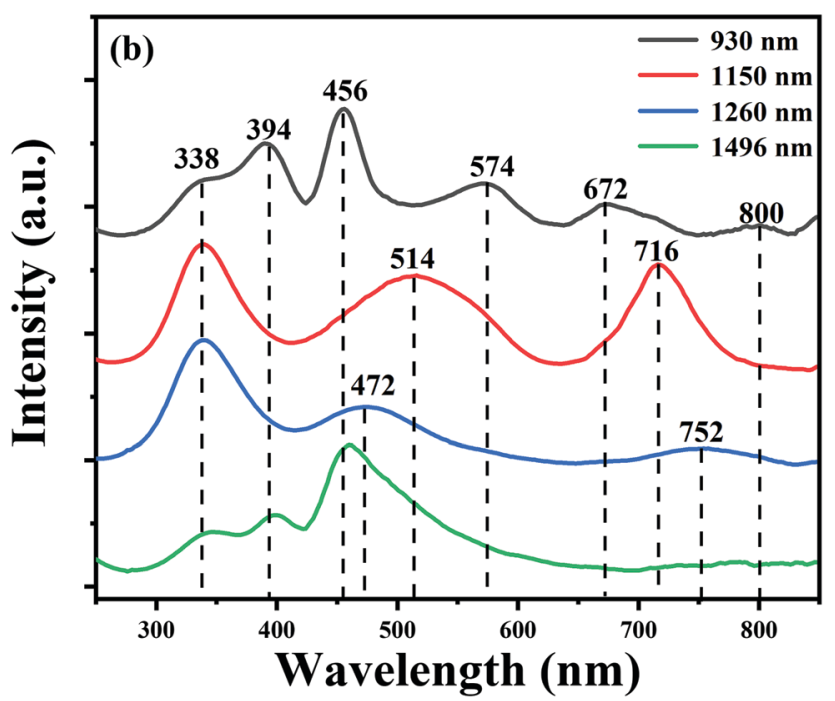

Fig. 4 Excitation spectra at 930, 1150, 1260 and $1490 \mathrm{~nm}$ for the BF sample. from the excitation bands located at $\sim 338, \sim 394$, and $\sim 456 \mathrm{~nm}$ for the $1490 \mathrm{~nm}$ emission band. Therefore, we inferred that the $\sim 924$ and $\sim 1496 \mathrm{~nm}$ emission bands may originate from different Bi-activated NIR centers. While monitoring at $\sim 1150 \mathrm{~nm}$, the excitation spectra consist of three strong bands locating at $\sim 338$, $\sim 514$ and $\sim 716 \mathrm{~nm}$, respectively. The $\sim 1260 \mathrm{~nm}$ emission band presents a strong absorption band at $\sim 338 \mathrm{~nm}$ and other broad excitation bands at $\sim 472$ and $\sim 752 \mathrm{~nm}$. The variation tendency observed in the excitation spectra can well explain the different behaviour of the multiple Bi-activated centers in some degree.

The fluorescence lifetime is another important parameter to characterize the fluorescence performance of Bi-doped glasses. The decay curves of multi-center NIR emission at 930, 1150, 1260 and $1490 \mathrm{~nm}$ under the excitation of $460 \mathrm{~nm}$ are presented in Fig. 5(a)-(d). All the decay curves followed a double exponential decaying law, as described by eqn (1):

$$
I(t)=A_{1} \exp \left(-t / \tau_{1}\right)+A_{2} \exp \left(-t / \tau_{2}\right)
$$

where $I(t)$ is the intensity at time $t ; \tau_{1}$ and $\tau_{2}$ are short and long decaying components, respectively; parameters $A_{1}$ and $A_{2}$ are fitting constants. Fitting each curve to the equation produced $\tau_{1}$ and $\tau_{2}$, and the correlation coefficients were always between 99.50\% and 99.94\%. As shown in Fig. 5, the obtained mean lifetimes for 930, 1150, 1260, and $1490 \mathrm{~nm}$ shared different responses with varying alkaline earth metals from $\mathrm{Mg}$ to $\mathrm{Ba}$. As listed in Table 1 , the mean lifetime for $\sim 930$ and $\sim 1490 \mathrm{~nm}$ was extended slightly, and that for $\sim 1260 \mathrm{~nm}$ significantly increased. However, the opposite trend was observed in the lifetimes of the emission at $1150 \mathrm{~nm}$. The opposite variation trends between $\sim 1150$ and $\sim 1260 \mathrm{~nm}$ gave further evidence that the energy has transferred from $\mathrm{Bi}^{+}$to $\mathrm{Bi}^{0}$. Moreover, compared to oxide glasses, Bi-doped oxyfluorogermanate glass with a low phono energy could reduce the probability of the nonradiative relaxation of Bi-activated NIR centers, thus obtaining a relatively long lifetime. ${ }^{22,39}$

In consideration of the above-mentioned results, here, proper energy level diagrams for $\mathrm{Bi}^{+}$and $\mathrm{Bi}^{0}$ were depicted on the basis of previous studies to explain the energy transfer mechanism. ${ }^{18,26}$ As shown in Fig. 6, both $\mathrm{Bi}^{+}$and $\mathrm{Bi}^{0}$ can be excited by $460 \mathrm{~nm}$ light from the ground state to the excited levels of ${ }^{1} \mathrm{D}_{2}$ and ${ }^{2} \mathrm{P}_{1 / 2}$, respectively, and the active $\mathrm{Bi}^{+}$then relaxes nonradiatively to the first excited level ${ }^{3} \mathrm{P}_{1}$. The energy transfer process can be described as ${ }^{3} \mathrm{P}_{1}\left(\mathrm{Bi}^{+}\right)+{ }^{4} \mathrm{~S}_{3 / 2}\left(\mathrm{Bi}^{0}\right) \rightarrow{ }^{3} \mathrm{P}_{0}\left(\mathrm{Bi}^{+}\right)+$ ${ }^{2} \mathrm{D}_{3 / 2}\left(\mathrm{Bi}^{0}\right)$, which helps to emit a $1260 \mathrm{~nm}$ photon. In order to quantify the energy transfer, we calculated the efficiency in $\mathrm{CF}$, $\mathrm{SF}$, and BF glasses compared to the MF sample by the following eqn (2):

$$
\mu_{\mathrm{Bi}^{+} \rightarrow \mathrm{Bi}^{0}}=1-\frac{\tau_{1150}}{\tau_{1150_{\mathrm{MF}}}}
$$

where $\mu_{\mathrm{Bi}^{+} \rightarrow \mathrm{Bi}^{0}}$ is the energy transfer efficiency from $\mathrm{Bi}^{+}$to $\mathrm{Bi}^{0}$; $\tau_{1150}$ is the mean lifetime of $\mathrm{Bi}^{+}$at the $1150 \mathrm{~nm}$ emission in $\mathrm{CF}, \mathrm{SF}$ and $\mathrm{BF}$ glasses; $\tau_{1150_{\mathrm{MF}}}$ is to the mean lifetime of $\mathrm{Bi}^{+}$at the $1150 \mathrm{~nm}$ emission in the MF glass. As listed in Table 1, the energy transfer efficiency increased from $1.61 \%$ to $5.62 \%$ with varying $\mathrm{CF}$ to $\mathrm{BF}$ glasses compared to MF glass. 

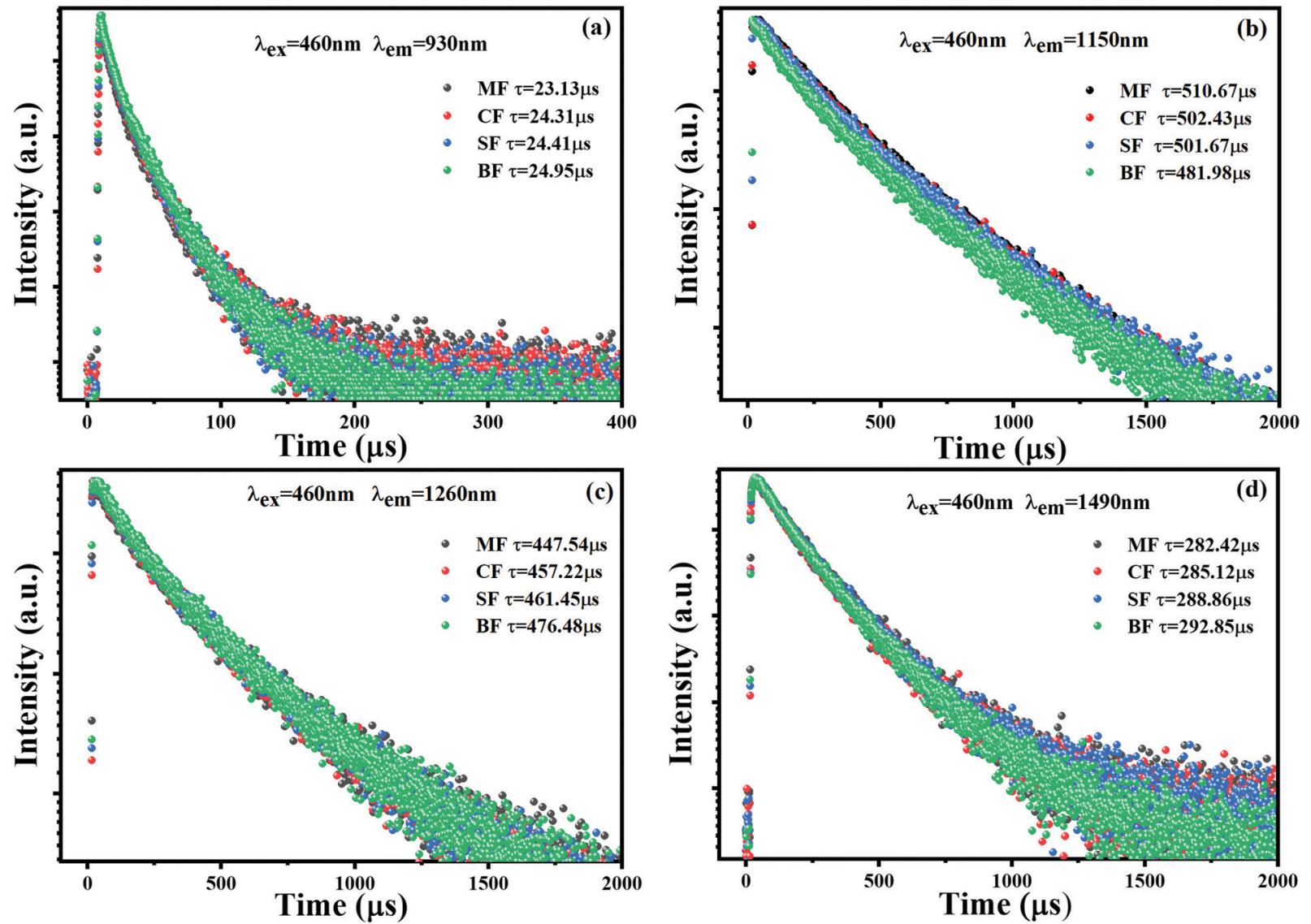

Fig. 5 Decaying curves of (a) 930, (b) 1150, (c) 1260, and (d) $1490 \mathrm{~nm}$ emission of MF, CF, SF, and BF glass samples $\left(\lambda_{\mathrm{ex}}=460 \mathrm{~nm}\right.$ ).

Table 1 The mean lifetime of $\mathrm{Bi}$ at $930,1150,1260$, and $1490 \mathrm{~nm}$, the energy transfer efficiency from $\mathrm{Bi}^{+}$to $\mathrm{Bi}^{0}$ in $\mathrm{CF}, \mathrm{SF}$, and $\mathrm{BF}$ glasses as compared to MF glass, and the values of the optical basicity in the glass samples

\begin{tabular}{lllllll}
\hline $\begin{array}{l}\text { Glass } \\
\text { samples }\end{array}$ & $\begin{array}{l}\tau_{930} \\
(\mu \mathrm{s})\end{array}$ & $\begin{array}{l}\tau_{1150}(\mu \mathrm{s}) \\
\mathrm{Bi}^{+}\end{array}$ & $\begin{array}{l}\tau_{1260}(\mu \mathrm{s}) \\
\mathrm{Bi}^{\circ}\end{array}$ & $\begin{array}{l}\tau_{1490} \\
(\mu \mathrm{s})\end{array}$ & $\begin{array}{l}\mu_{\mathrm{Bi}^{+} \rightarrow \mathrm{Bi}^{0}} \\
(\%)\end{array}$ & $\begin{array}{l}\text { Optical } \\
\text { basicity }\left(\Lambda_{\mathrm{th}}\right)\end{array}$ \\
\hline $\mathrm{MF}$ & 23.13 & 510.67 & 447.54 & 282.42 & - & 0.601 \\
$\mathrm{CF}$ & 24.31 & 502.43 & 457.22 & 285.12 & 1.61 & 0.606 \\
$\mathrm{SF}$ & 24.41 & 501.67 & 461.45 & 288.86 & 1.76 & 0.609 \\
$\mathrm{BF}$ & 24.95 & 481.98 & 476.48 & 292.85 & 5.62 & 0.611 \\
\hline
\end{tabular}

Owing to the co-existence of multiple Bi-activated NIR centers in the glasses, the ultrabroadband NIR emission in Bi-doped germanate glasses prepared through topochemical reduction could be tuned widely to span the NIR region by adjusting the excitation wavelength from 420 to $700 \mathrm{~nm}$, as presented in Fig. 7(a). Compared to RE-doped or RE-Bi co-doped glasses, the NIR emission performance of Bi-doped glasses was more flexible and efficient in terms of the emission bandwidth and peaks. ${ }^{40,41}$ To further reveal the relationship between the emission and excitation behaviour in detail, the emission-excitation map from the $\mathrm{BF}$ glass sample is denoted in Fig. 7(b). Clearly, there are multiple NIR centers co-existing in the glass and Bi-activated centers responded differently to the excitation wavelength. For NIR emission centers at $\sim 1100 \mathrm{~nm}$, two wide absorption bands

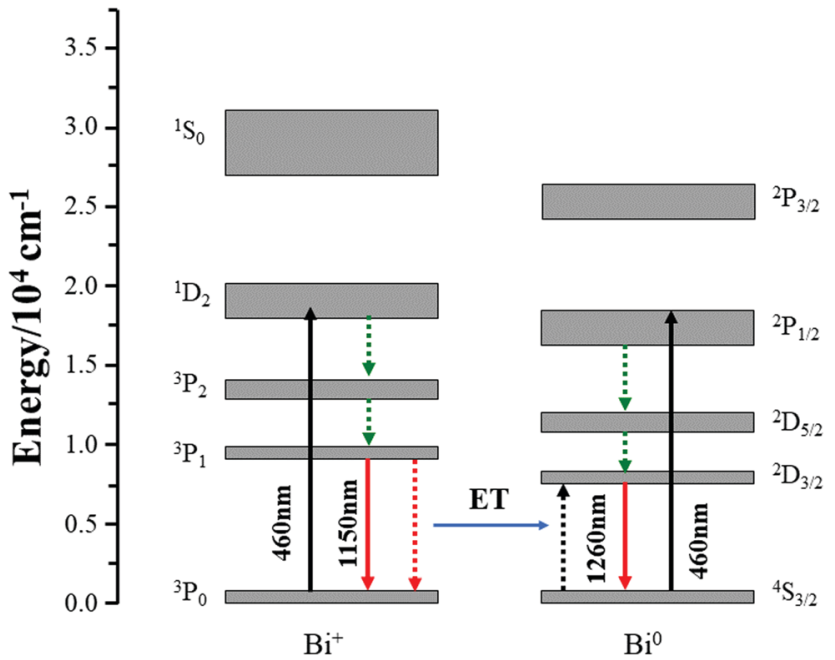

Fig. 6 Simplified energy level diagram of $\mathrm{Bi}^{+}$and $\mathrm{Bi}^{0}$, and the possible energy transfer between $\mathrm{Bi}^{+}$and $\mathrm{Bi}^{\circ}$.

ranging from 420 to $600 \mathrm{~nm}$ and 660 to $720 \mathrm{~nm}$ are observed, which indicates that the emission band around $\sim 1100 \mathrm{~nm}$ could be excited by visible light. For the NIR emissions centered at $\sim 930$ and $\sim 1500 \mathrm{~nm}$, there was only a narrow absorption band between 430 and $470 \mathrm{~nm}$. By increasing the excitation wavelength 

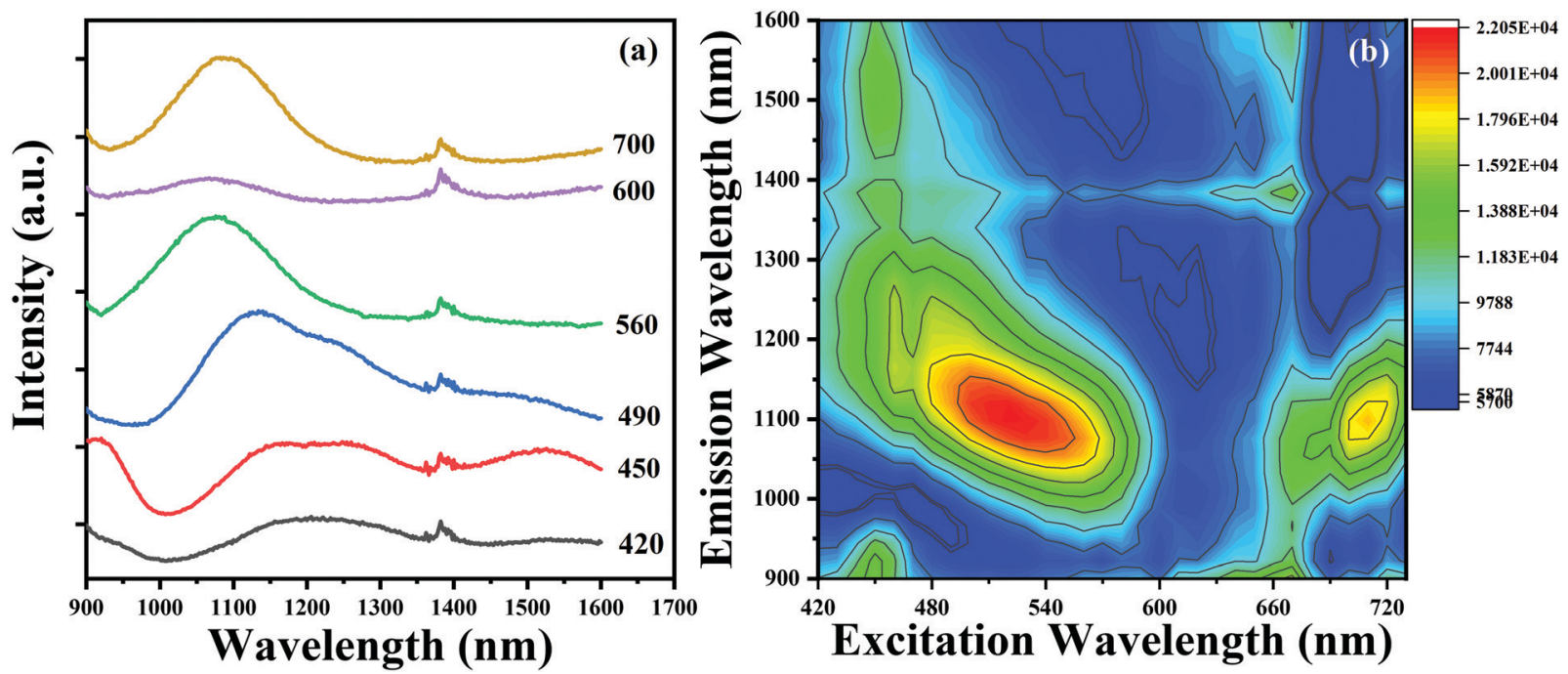

Fig. 7 (a) The NIR spectra evolution of the BF glass sample. (b) The contour plot of the emission-excitation map from the BF glass sample.

from 420 to $720 \mathrm{~nm}$, the emission band can cover the 900-1600 nm region at the beginning, but set to $\sim 1100 \mathrm{~nm}$ at the end. These results provided a flexible pumping scheme of Bi-doped glasses for the potential application as NIR materials.

In order to understand why the above changes occurred, the concept of optical basicity, which has an important influence on the valence state and luminescence behaviour of $\mathrm{Bi}$, was employed to provide more clues. ${ }^{37,38}$ The values of the Duffy's optical basicity $\left(\Lambda_{\mathrm{th}}\right)$ of glasses can be calculated by the eqn $(3)$ :

$$
\Lambda_{\mathrm{th}}=\left(X_{1} \Lambda_{1}+X_{2} \Lambda_{2}+\cdots+X_{n} \Lambda_{n}\right)
$$

where $\Lambda_{n}$ is the optical basicity values of each composition of glass, and $X_{n}$ is the equivalent fraction of the oxides and the fluorides that composition $n$ contributes. Values of the optical basicity $\Lambda_{n}$ can be decided by referring to ref. 42 . As presented in Table 1 , the optical basicity values of glasses increased from 0.600 to 0.611 for the samples varying from MF to BF. It is widely accepted that the low optical basicity favors Bi ions with a low valence state, while higher ones exist easily in glasses with a higher optical basicity. ${ }^{32,37}$ Increasing the optical basicity values of the glass will unavoidably change the local environment around $\mathrm{Bi}$-activated centers, thus triggering the chain reaction of $\mathrm{Bi}$ clusters or metal particles: $\mathrm{Bi}^{0} \rightarrow \mathrm{Bi}^{+} \rightarrow \mathrm{Bi}^{2+} \rightarrow \mathrm{Bi}^{3+}$, resulting in a diversity of luminescence behaviours.

To the best of our knowledge, the Bi-activated centers are highly sensitive to the variation of the local crystal field and glass structures around them. ${ }^{43-45}$ To reveal the deeper mechanism behind what we have discussed above, the structural analysis by FTIR spectroscopy and EPR was performed to offer more detailed information concerning the relationship between the microstructure of the glasses and the optical properties of Bi-activated NIR centers. FTIR spectra are illustrated in Fig. 8. Two absorption bands around $578 \mathrm{~cm}^{-1}$ and $862 \mathrm{~cm}^{-1}$ are observed in the spectra, which are assigned to the symmetric stretching vibrations of $\mathrm{Ge}-\mathrm{O}-\mathrm{Ge}$ and the asymmetric stretching vibrations of $\mathrm{Ge}-\mathrm{O}-\mathrm{Ge}$ in the $\mathrm{GeO}_{4}$ tetrahedra. ${ }^{24,37,46}$ As the radius of the alkaline earth-
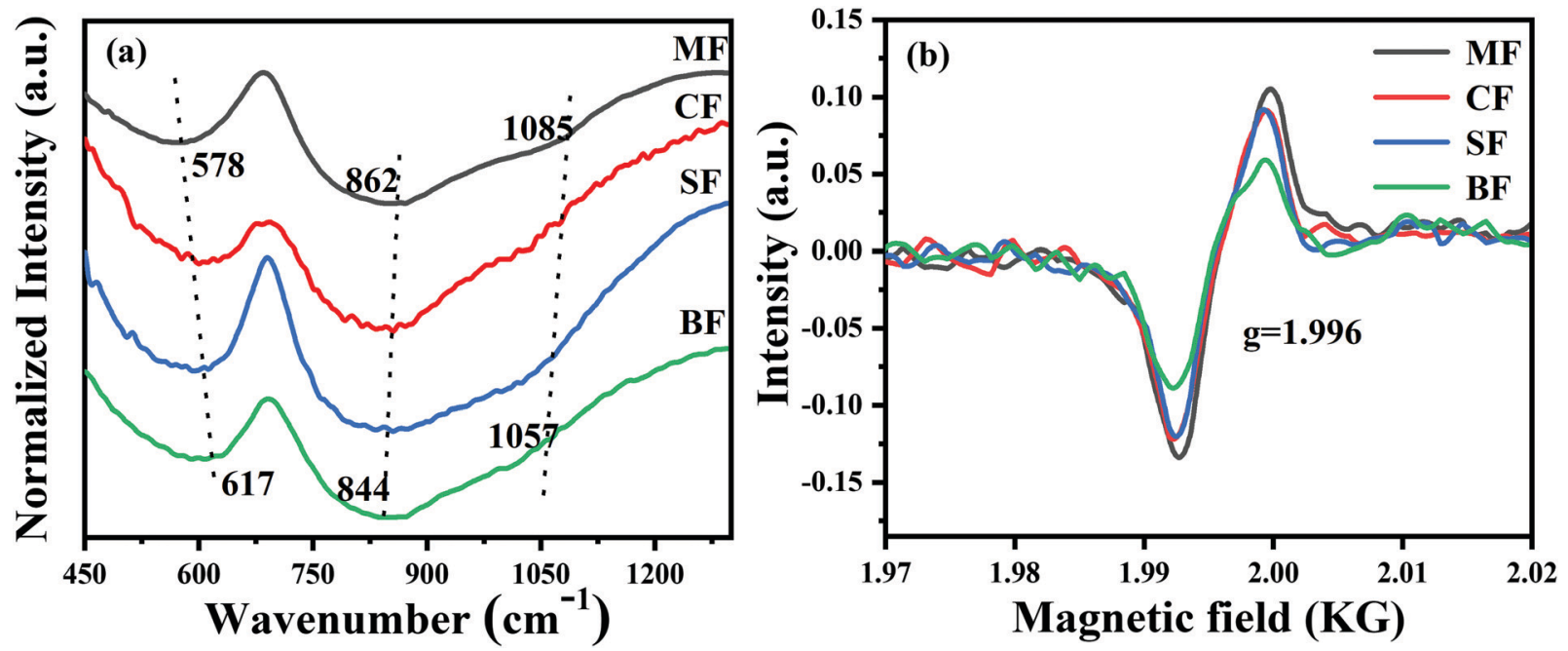

Fig. 8 (a) Normalized FTIR spectra and (b) EPR spectra of MF, CF, SF, BF glass samples. 


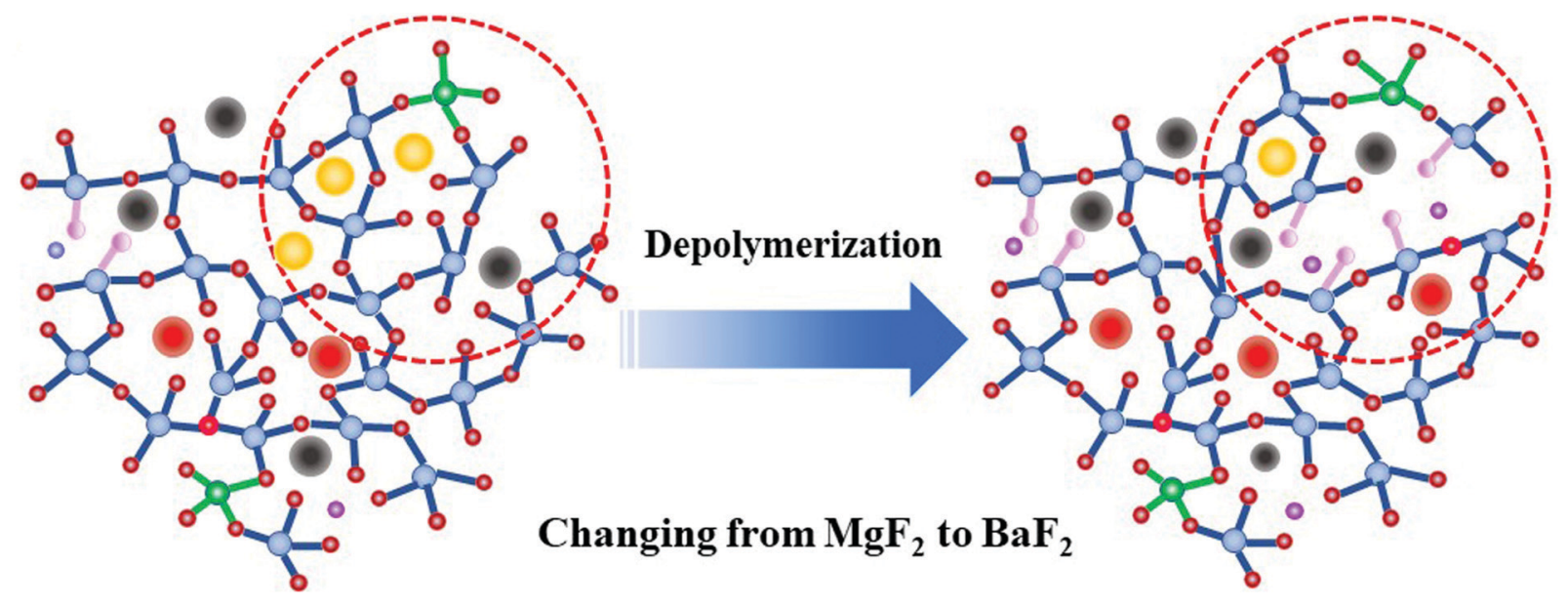

\section{Active Bi $\odot$ New active Bi $\odot$ Inactive Bi $\bigcirc$ Ge $\odot \mathrm{Al} \odot \mathbf{O} \bullet \mathbf{N} \odot \mathbf{F} \odot \mathbf{B a} \odot \mathbf{M g}$}

Fig. 9 Evolution of the microscopic structure of the glasses MF to BF.

metal ions increases, two remarkable changes are observed in the FTIR spectra. First, the former absorption peak located at $578 \mathrm{~cm}^{-1}$ ultimately shifted to $617 \mathrm{~cm}^{-1}$, indicating that the sixunit $\mathrm{GeO}_{4}$ rings are broken up into smaller ones, and eventually, three-unit $\mathrm{GeO}_{4}$ rings. Second, the latter absorption peaks blueshift from $862 \mathrm{~cm}^{-1}$ to $844 \mathrm{~cm}^{-1}$ and from $1085 \mathrm{~cm}^{-1}$ to $1057 \mathrm{~cm}^{-1}$, which are related to the conversion of the $\mathrm{GeO}_{4}$ tetrahedra from $\mathrm{Q}^{3}$ (tetrahedral with one non-bridged oxygen) to

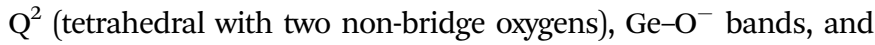
finally forming $\mathrm{GeO}_{6}$ octahedron. ${ }^{47-49}$ To sum up, the phenomenon observed above implies that more and more non-bridging oxygens are generated in the glasses, which therefore resulted in the depolymerisation of the glass network. The EPR technique is adopted to offer more information about the glass structure. As presented in Fig. 8(b), an obvious signal at $g=1.996$ appeared in the four samples, which is attributed to the oxygen vacancy. Moreover, the signal intensity decreases continuously with the increase in the radius of the alkaline earth metal ions, indicating that more and more oxygen were involved into the glass structures, thus causing the Bi-activated NIR centers to be oxidized into non-NIR emission centers. This fact shows that the NIR luminescence behaviour of Bi could be regulated by modulating the overall glass structure.

Based on the above analysis, the structural evolution of the glasses from MF to BF is proposed in the schematic diagram in Fig. 9. As the ionic field strength decreases from $\mathrm{Mg}^{2+}$ to $\mathrm{Ba}^{2+}$, more and more $\mathrm{F}^{-}$replace $\mathrm{O}^{2-}$ with varying alkaline earth metal fluorides from $\mathrm{MgF}_{2}$ to $\mathrm{BaF}_{2}$, which results in an increase in the content of non-bridging oxygen. As a result, the glass networks are gradually depolymerized. On one hand, it creates a negatively charged environment, which requires more positive charges to neutralize the excess negative charges, and the looser glass structures can easily expose the Bi NIR emission centers within the glasses to the oxygen atmosphere. ${ }^{21}$ The above two reasons drive the oxidation of $\mathrm{Bi}$ in low valence into higher valence, which are not active NIR centers. ${ }^{50-52}$ Simultaneously, the depolymerized glass network promotes interactions between $\mathrm{Bi}^{+}$ and $\mathrm{Bi}^{0}$. As a result, the energy transfer between $\mathrm{Bi}^{+}$and $\mathrm{Bi}^{0}$ is enhanced, which is why we could observe the increased energy transfer efficiency from $\mathrm{Bi}^{+}$to $\mathrm{Bi}^{0}$ in Table 1 . On the other hand, the depolymerized glass network may facilitate the formation of more nitride bonds connecting with Ge to the glass network around $\mathrm{Bi}$, thus generating more new Bi NIR emission centers related to germanate by nitridation. ${ }^{33}$ As a consequence, the emission intensity at $\sim 924$ and $\sim 1496 \mathrm{~nm}$ improved. This result indicates that the multi-center Bi NIR luminescence behaviour can be regulated differently by adjusting the glass structures, and provides a better understanding about the mechanism of the Bi NIR emission.

\section{Conclusions}

In summary, through adjusting the glass structure, we successfully manipulated the multi-center Bi NIR luminescence behaviours in fluorine- and nitrogen-doped germanate glasses. A flatter emission spectrum spanning from 850 to $1600 \mathrm{~nm}$ with predominant emission bands located at $\sim 924, \sim 1150, \sim 1260$ and $\sim 1496 \mathrm{~nm}$ was realized. By increasing the radius of alkaline earth metal ions, the glass structures were gradually depolymerized. The looser glass structures, on one hand, promoted the oxidization of $\mathrm{Bi}^{0}$ and $\mathrm{Bi}^{+}$ into inactive centers, thus reducing the emission intensity at $\sim 1150 \mathrm{~nm}$. On the other hand, it enhanced the energy transfer from $\mathrm{Bi}^{+}$to $\mathrm{Bi}^{0}$, which was conducive to enhance the emission intensity at $\sim 1260 \mathrm{~nm}$. Moreover, the depolymerized glass network may facilitate the formation of more nitride bonds connecting to Ge to the glass network around $\mathrm{Bi}$, thus generating new Bi NIR emission centers related to germanate by nitridation. As a result, the emission intensity and lifetime of $\sim 924$ and $\sim 1496 \mathrm{~nm}$ emission bands got a certain degree of improvement. The study presented here is expected to promote our understanding to the mechanism of the Bi NIR emission behaviours in glass. 


\section{Conflicts of interest}

There are no conflicts to declare.

\section{Acknowledgements}

We acknowledge the financial support from the National Key R\&D Program of China (2020YFB1805901), the National Natural Science Foundation of China (Grant No. 51872095, 51672085), the Key R\&D Program of Guangzhou (No. 202007020003), the Program for Innovative Research Team in University of Ministry of Education of China (Grant No. IRT-17R38), the Joint Fund of Ministry of Education of China, the Major Basic Research Cultivation Project of Natural Science Foundation of Guangdong Province (Grant No. 2018B03038009), the Local Innovative Research Team Project of "Pearl River Talent Plan" (Grant No. 2017BT01X137) and the Fundamental Research Funds for the Central Universities (Grant No. 2020ZYGXZR036).

\section{References}

1 S. A. Diddams, L. Hollberg and V. Mbele, Nature, 2007, 445, 627-630.

2 B. Lee, Opt. Fiber Technol., 2003, 9, 57-79.

3 Th. Udem, R. Holzwarth and T. W. Hänsch, Nature, 2002, 416, 233-237.

4 M. Hilbert and P. López, Science, 2011, 332, 60-65.

5 M. Janos and S. C. Guy, J. Lightwave Technol., 1998, 16, 542-548.

6 S. Jetschke and S. Unger, et al., Opt. Express, 2008, 16, 15540-15545.

7 R. Zhou, Y. Ju, Y. Zhang and Y. Wang, Chin. Opt. Lett., 2011, 9, 071401.

8 X. Liu, P. Kuan, D. Li, S. Gao, X. Wang, L. Zhang, L. Hu and D. Chen, Opt. Mater. Express, 2016, 6, 1093-1098.

9 R. Lisiecki and W. Ryba-Romanowski, J. Alloys Compd., 2020, 814, 152304.

10 K. Murata, Y. Fujimoto, T. Kanabe, H. Fujita and M. Nakatsuka, Fusion Eng. Des., 1999, 44, 437-439.

11 Y. Fujimoto and M. Nakatsuka, Jpn. J. Appl. Phys., 2001, 40, L279-L281.

12 M. Peng, J. Qiu, D. Chen, X. Meng, I. Yang, X. Jiang and C. Zhu, Opt. Lett., 2004, 29, 1998-2000.

13 E. M. Dianov, V. V. Dvoyrin, V. M. Mashinsky, A. A. Umnikov, M. V. Yashkov and A. N. Gur'yanov, Quantum Electron., 2005, 35, 1083-1084.

14 Y. Fujimoto and M. Nakatsuka, J. Non-Cryst. Solids, 2006, 352, 2254-2258.

15 Y. Arai, T. Suzuki, Y. Ohishi, S. Morimoto and S. Khonthon, Appl. Phys. Lett., 2007, 90, 261110.

16 G. Lakshminarayana, J. Ruan and J. Qiu, J. Alloys Compd., 2009, 476, 878-883.

17 M. A. Hughes, T. Akada, T. Suzuki, Y. Ohishi and D. W. Hewak, Opt. Express, 2009, 17, 19345-19355.

18 N. Zhang, J. Qiu, G. Dong, Z. Yang, Q. Zhang and M. Peng, J. Mater. Chem., 2012, 22, 3154-3159.
19 Y. Zhao, M. Peng, A. Mermet, J. Zheng and J. Qiu, J. Mater. Chem. C, 2014, 2, 7830-7835.

20 J. Cao, L. Wondraczek, Y. Wang, L. Wang, J. Li, S. Xu and M. Peng, ACS Photonics, 2018, 5, 4393-4401.

21 X. Li, J. Cao, L. Wang and M. Peng, J. Am. Ceram. Soc., 2018, 101, 1159-1168.

22 J. Cao, J. Peng, L. Wang, H. Luo, X. Wang, P. Xiong, Y. Wang and M. Peng, J. Mater. Chem. C, 2019, 7, 2076-2084.

23 J. Cao, X. Li, L. Wang, Z. Zhang, S. Xu and M. Peng, J. Mater. Chem. C, 2018, 101, 2297-2304.

24 J. Cao, S. Xu, Q. Zhang, Z. Yang and M. Peng, Adv. Opt. Mater., 2018, 6, 1801059.

25 B. Xu, S. Zhou, M. Guan and D. Tan, Opt. Express, 2011, 19, 23436-23443.

26 L. Zhang, G. Dong, J. Wu, M. Peng and J. Qiu, J. Alloys Compd., 2012, 531, 10-13.

27 F. E. D. Zhang, R. Ye, Y. Hua, S. Xu and F. Huang, Opt. Mater., 2019, 95, 109222.

28 S. Wu, P. Xiong, X. Liu, Y. Fu, Q. Liu, M. Peng, Y. Chen and Z. Ma, J. Mater. Chem. C, 2020, 8, 16584-16592.

29 X. Xu, J. Zhao, X. Chen, Q. Xu, T. Wang, S. F. Yu, X. Xu, X. Qiao, J. Du, X. Fan, J. Qiu and G. Qian, J. Am. Ceram. Soc., 2020, 103, 5796-5807.

30 A. J. Stevenson, H. Serier-Brault, P. Gredin and M. Mortier, J. Fluorine Chem., 2011, 132, 1165-1173.

31 Y. Ma, X. Peng, M. Fei, W. Zhang, L. Teng, F. Hu, R. Wei and H. Guo, J. Alloys Compd., 2020, 846, 156435.

32 B. Xu, D. Tan, S. Zhou, Z. Hong, K. N. Sharafudeen and J. Qiu, Opt. Express, 2012, 20, 29105-29111.

33 W. Chen, J. Cao, M. Peng, Y. Wang and P. Xiong, J. Am. Ceram. Soc., 2020, 00, 1-9.

34 J. Cao, L. Li, L. Wang, X. Li, Z. Zhang, S. Xu and M. Peng, J. Mater. Chem. C, 2018, 6, 5384-5390.

35 X. Li, J. Cao and M. Peng, J. Am. Ceram. Soc., 2018, 101, 2921-2929.

36 C. Liu, Y. Zhuang, J. Han, J. Ruan, C. Liu and X. Zhao, Ceram. Int., 2020, 46, 15544-15553.

37 Y. Zhao, L. Wondraczek, A. Mermet, M. Peng, Q. Zhang and J. Qiu, Opt. Express, 2015, 23, 12423-12433.

38 Y. Xue, J. Cao, Z. Zhang, L. Wang, S. Xu and M. Peng, J. Am. Ceram. Soc., 2018, 101, 624-633.

39 X. Li, F. Hu, M. Peng, Q. Zhang and M. Yin, J. Am. Ceram. Soc., 2017, 100, 574-582.

40 J. Xiao, J. Cao, Y. Wang, X. Li, X. Wang, J. Zhang and M. Peng, J. Mater. Chem. C, 2019, 7, 10544-10550.

41 H. K. Dan, D. N. Le, H. T. Nguyen-Truong, T. D. Tap, H. X. Vinh, N. M. Ty, R. Wang, D. Zhou and J. Qiu, J. Lumin., 2020, 219, 116942.

42 J. A. Duffy, J. Non-Cryst. Solids, 1989, 109, 35-39.

43 P. Xiong, Y. Li and M. Peng, iScience, 2020, 23, 101578.

44 Z. Lu, W. Zhang, J. Chen, S. Chen, J. Cao and H. Guo, J. Lumin., 2021, 232, 117857.

45 Z. Zheng, J. Zhang, X. Liu, R. Wei, F. Hu and H. Guo, Ceram. Int., 2020, 46, 6154-6159.

46 H. Verweij and J. H. J. M. Buster, J. Non-Cryst. Solids, 1979, 34, 81-99. 
47 F. L. Galeener, A. E. Geissberger, G. W. Ogar and R. E. Loehman, Phys. Rev. B: Condens. Matter Mater. Phys., 1983, 28, 4768-4773.

48 D. D. Martino, L. F. Santos, A. C. Marques and R. M. Almeida, J. Non-Cryst. Solids, 2001, 293-295, 394-401.

49 L. Wang, M. Peng, X. Li, Y. Wang, H. Luo, J. Cao and X. Li, J. Mater. Chem. C, 2019, 7, 5074-5083.
50 P. Xiong, C. Zheng, M. Peng, Z. Zhou, F. Xu, K. Qin, Y. Hong and Z. Ma, J. Am. Ceram. Soc., 2020, 103, 6922-6931.

51 C. Zheng, P. Xiong, M. Peng and H. Liu, J. Mater. Chem. C, 2020, 8, 13668-13675.

52 W. Zhang, Y. Tong, F. Hu, R. Wei, L. Chen and H. Guo, Ceram. Int., 2021, 47, 284-291. 